\title{
Optimization Of (s, S) Periodic Review Inventory Model With Uncertain Demand And Lead Time Using Simulation
}

Raida Abuizam, Purdue University Calumet, USA

\begin{abstract}
This paper presents the use of Palisade @RISK simulation and RISKOptimizer to minimize the expected cost of inventory per period over a long time horizon. An $(s, S)$ ordering policy will be used in this analysis. In an $(s, S)$ ordering policy, an order is placed at the beginning of any period in which beginning inventory is less than s. The order size is the amount needed to bring the inventory level up to S. This paper illustrates a comparison between the use of @RISK simulation with trial values of $(s, S)$ and the use of RISKOptimizer to find optimal values of $(s, S)$ that minimize the expected cost of inventory over a period of time in a periodic review inventory model.
\end{abstract}

Keywords: Inventory Management; Simulation; Periodic Review inventory model

\section{INTRODUCTION}

nventory management is an important concern for managers in all types of businesses. For companies that operate on relatively low profit margins, poor inventory management can seriously damage the business. The challenge is neither to bare inventories to the bone to reduce costs nor to have plenty around to satisfy all demands. Rather, an inventory manager's job is to balance the conflicting costs and pressures that argue for both low and high inventories and determine an appropriate inventory level there between. The balance is typically not easy to find, so managers need models to determine the best ordering policy.

An inventory problem can be broken up into two parts - how much to order and when to place an order. When customer demand is known and certain, managers can apply any of the deterministic inventory models that exist in the literature, such as the economic order quantity model. A more realistic situation occurs when customer demands are not certain. Another element that can provide uncertainty is the amount of lead time between placement and receipt of an order. Therefore, managers require models that deal with probabilistic inventory parameters.

Companies that face uncertainties in their inventory parameters have three basic alternative solutions. First, they can use best guesses for uncertain quantities and proceed with one of the deterministic models. Second, they can develop a mathematical model to deal with uncertainty. The disadvantage of this approach is that these analytical models can be very complex and difficult for many managers to comprehend. The third option to capture the uncertainty in the inventory parameters is to develop a simulation model. The advantage of the simulation model is that it is relatively easy to develop regardless of the complexity of the problem.

In this paper, a periodic review inventory system is presented using simulation. It describes a type of ordering policy called $(\mathrm{s}, \mathrm{S})$ that is commonly used in periodic review situations, where $\mathrm{s}$ is the reorder point and $\mathrm{S}$ is the order-up-to-quantity point. In the periodic review system, an item's inventory position is reviewed periodically, rather than continuously. This system can simplify delivery scheduling because it establishes a routine. A new order is placed at the end of each review, and the time between orders is fixed. Demand is a random variable, so total demand between reviews varies. In the periodic review system, the lot size $\mathrm{Q}$ may change from one order to another. Lead time is also uncertain; i.e., it may follow a probability distribution. This paper illustrates a comparison 
between the use of Palisade @RISK simulation with trial values of $(\mathrm{s}, \mathrm{S})$ and the use of Palisade RISKOptimizer to find optimal values of $(\mathrm{s}, \mathrm{S})$ that minimize the expected cost of inventory in a periodic review inventory model. RISKOptimizer combines the genetic algorithm technology of Evolver with the Monte Carlo simulation engine of @ RISK to solve optimization problems. It performs optimizations under uncertainty, finding the best combination of parameters while accounting for random, uncontrolled factors.

\section{LITERATURE REVIEW}

Barankin (1961), Daniel (1962), Neuts (1964), Bulinskaya (1964), Veinnot (1966), and Wright (1968) provided studies of the periodic inventory systems with deterministic lead time for regular and emergency ordering, where the regular order lead time is one period and the emergency replenishment is instantaneous. Another periodic review system has been studied by Gross and Soriano (1972). Gross and Soriano analyzed the decision of choosing which supply mode to use at a review opportunity. Whittemore and Saunders (1977) derived an optimal ordering policy using a complicated multi-sate dynamic program. They obtained explicit results if the regular and emergency lead times differed by one period.

Tijms and Groenevelt (1984) considered a general class of (s, S) models, covering both periodic and continuous review cases. They allowed random lead time while prohibiting order crossing. They compared their proposed approximation with optimal results obtained from Lagrangian methods. Blumenfield et al. (1985) allowed continuous emergency ordering, and assumed that the fixed size of the instantaneous emergency order was large enough to avoid any stock-out until the next regular order arrives.

Schneider and Ringuest (1990) considered a periodic review system operating under a $\gamma$-service level measure, where ( 1 - $\gamma$ was the fraction of demand on backorder each period. They constructed a Lagrangian function, and proposed an algorithm that gave nearly exact solutions to the first order conditions. However, they assumed a fixed regular lead time.

The study of Chiang and Gutierrez (1996) was the first one that considered lead time, which can be shorter than the review period. In a later study, Chiang and Gutierrez (1998) allowed multiple emergency orders to be placed at any time within the review period. They developed a stochastic dynamic program to obtain the order-up-to level policy. They proved that the emergency order -up-to level increased in the number of periods until the next regular order arrived. The model became quite complex especially if the two lead times differed by more than one time unit.

On the other hand, Tagaras and Vlachos (2001) only allowed one emergency order per review period. The plan of the emergency order was to arrive one time unit before the regular order arrived, when the likelihood of a stock-out was highest. They developed an approximate cost model with respect to order-up-to level parameters. Their proposed model offered significant cost savings relative to other systems that did not allow emergency replenishment as an option.

Lee and Nahmias (1993) developed a cost minimization problem that minimized the cost of inventory incurred in terms of setup and holding cost. The model solved for an (s, S) pair that minimized inventory cost subject to the constraint that the solution satisfied a prescribed level of customer service. The model assumed that $S$ $\mathrm{s}$ is sufficiently large compared to the average period demand $\mu_{\mathrm{d}}$, where $\mathrm{S}-\mathrm{s} \geq 1.5 \mu_{\mathrm{d}}$.

Bashyam and $\mathrm{Fu}(1998)$ considered a periodic review (s, S) inventory system with random lead times and allowed for orders to cross in time. They adopted the "fill-rate" service level measured by Tim and Groenevelt. Their objective was to determine values of $s$ and $\mathrm{Q}=\mathrm{S}-\mathrm{s}$ that minimized the average setup and holding cost per period, subject to the constraint that the fill-rate was above a prescribed level. In their study, they proposed a feasible directions procedure that was simulation-based. They also presented computational results for a large number of test cases.

This study provides a simulation-based model that allows managers to combine assumptions about uncertain demand and ordering policies and then play out the events as they occur through time. This paper 
illustrates a comparison between the use of Palisade @RISK simulation with trial values of (s, S) and the use of Palisade RISKOptimizer to find optimal values of $(s, S)$ that will minimize inventory cost. The objective of this approach is to provide inventory managers with a spreadsheet model that deals with different types of probability distributions of demand and behavior of the lead time. If demand in any period is not satisfied from on-hand inventory, then demand is met by an instantaneous emergency order at a higher cost.

Using simulation to manage inventory with uncertain demand and lead time is not simple. It requires a good understanding of Excel functions and how to apply them to support one's argument and reasoning. However, an analytical (non-simulation) model with many uncertainties is very complex and will be out of the question for many managers, unless they are mathematically knowledgeable experts. The spreadsheet model in this paper will provide managers with a technique to use in order to minimize total inventory cost. It will also allow managers to make changes to the model as the changes occur. The major benefit of this model is that it does not require managers to have mathematical skills to apply it.

\section{SPREADSHEET SIMULATION MODEL DEVELOPMENT}

There are three elements required in building any spreadsheet simulation model. The first element is that managers need a method to enter random quantities from particular probability distributions in input cells. At least one of the input variable cells in a simulation model should contain random numbers. The second element is to use the right type of Excel formulas to relate the output cells to the inputs. The third and most important element in simulation spreadsheet modeling is the ability to make the spreadsheet recalculate many times and capture the resulting outputs for statistical analysis.

The selected input distributions for any simulation model reflect historical data and an analyst's best judgment as to what probability distribution best fit these data. In general, a probability distribution indicates the possible values of a random variable and the probabilities of these values. There are many probability distributions than can be used in any simulation model. Some of them are discrete, others are continuous. Modelers should always attempt to choose an appropriate distribution for each specific problem. Palisade @RISK provides a number of functions for simulating from various probability distributions. @RISK has many tools that help modelers to perform all the steps needed in any simulation model.

In this study, the use of Palisade @RISK simulation with trial values of $(\mathrm{s}, \mathrm{S})$ is illustrated in a spreadsheet model in order to minimize the expected total cost of inventory per period over a long time horizon. Palisade @RISK has a special function, RISKSIMTABLE, which allows modelers to run the same simulation several times using a different value of some key input variable each time. In this study, the key input variable is the values of (s, $\mathrm{S})$. A spreadsheet simulation model is developed to solve for (s, $\mathrm{S}$ ), where $\mathrm{s}$ is the reorder point, $\mathrm{S}$ is the order- upto quantity point, and s should be less than S. If the inventory position of an item at the beginning of the review period is at level $\mathrm{x}$, and $\mathrm{x}$ is less than or equal to $\mathrm{s}$, then the company will order exactly (S-x) items to bring the inventory level up to $\mathrm{S}$. Otherwise, if the inventory level is greater than $\mathrm{s}$, then no order is placed for that review period. If demand cannot be satisfied completely from an on-hand inventory, then an emergency order is placed at the end of the review period for the shortage. The model assumes that this order will arrive instantaneously at a penalty cost.

Palisade @RISK provides several options for displaying results. The big questions are which results the modelers want and where they want them. For a quick report, modelers can choose summary statistics measures. This report shows the summary for all designated inputs and outputs. This result summary window will also show a histogram for each output. @RISK can also provide detailed statistics reports for the simulation results that can be stored permanently in an Excel worksheet.

In this paper, two numerical examples will be presented to explain the methodology used in the spreadsheet model. For simplicity, both examples will have the same inputs. The only difference between the two examples will be the behavior of the demand. The first example will demonstrate a demand that is approximated by a discrete probability distribution while the second example will display a demand that is predicted by a normal probability distribution. The reason for these choices for the demand behavior is their common use in real business applications. 
A periodic review inventory model of fifty-two weeks will be studied for the two examples. A discussion and comparison of the results will follow.

To develop the spreadsheet model, the following input information is collected:

- Various costs (order cost, unit cost, holding cost per unit per period, and emergency shipment cost).

- The approximation of the probability distribution of the demand.

- $\quad$ Current inventory situation.

- $\quad$ The approximation of the probability distribution of the lead time.

- $\quad$ Possible trial values of s (reorder point) and S (order up to quantity point) to examine.

\section{NUMERICAL EXAMPLE 1}

Ray's Appliances is a retail store that has to place an order for one of its famous cordless mini drills. The fixed cost of placing an order is $\$ 300$ regardless of the size of the order. The unit cost is $\$ 15$ and the estimate of holding a drill in inventory for one week is $\$ 2$. Ray's Appliances has an initial inventory of 800 units on hand. No orders are due for the following two weeks. The order lead time is uncertain. Ray's manager estimates that if an order is placed, it will arrive after a lead time of one, two or three weeks with probabilities $0.5,0.3$, and 0.2 respectively. The weekly demand for the mini drills is also uncertain. Based on historical data, Ray's Appliances manager estimates that the weekly demand for the cordless mini drill will be 300, 400, 500, 600, or 700 with respective probabilities $0.1,0.2,0.25,0.3$, and 0.15 . If weekly demand is not met by on hand inventory, then an emergency order is placed at a high cost of $\$ 25$.

Ray's Appliances manager is planning to use Palisade @RISK simulation to simulate ten combinations of $(\mathrm{s}, \mathrm{S})$ ordering policy in order to determine which combination provides the lowest cost of inventory over the 52week period. Exhibit 1 presents Ray's Appliances inputs used in the spreadsheet model including the ten trial values of $(\mathrm{s}, \mathrm{S})$.

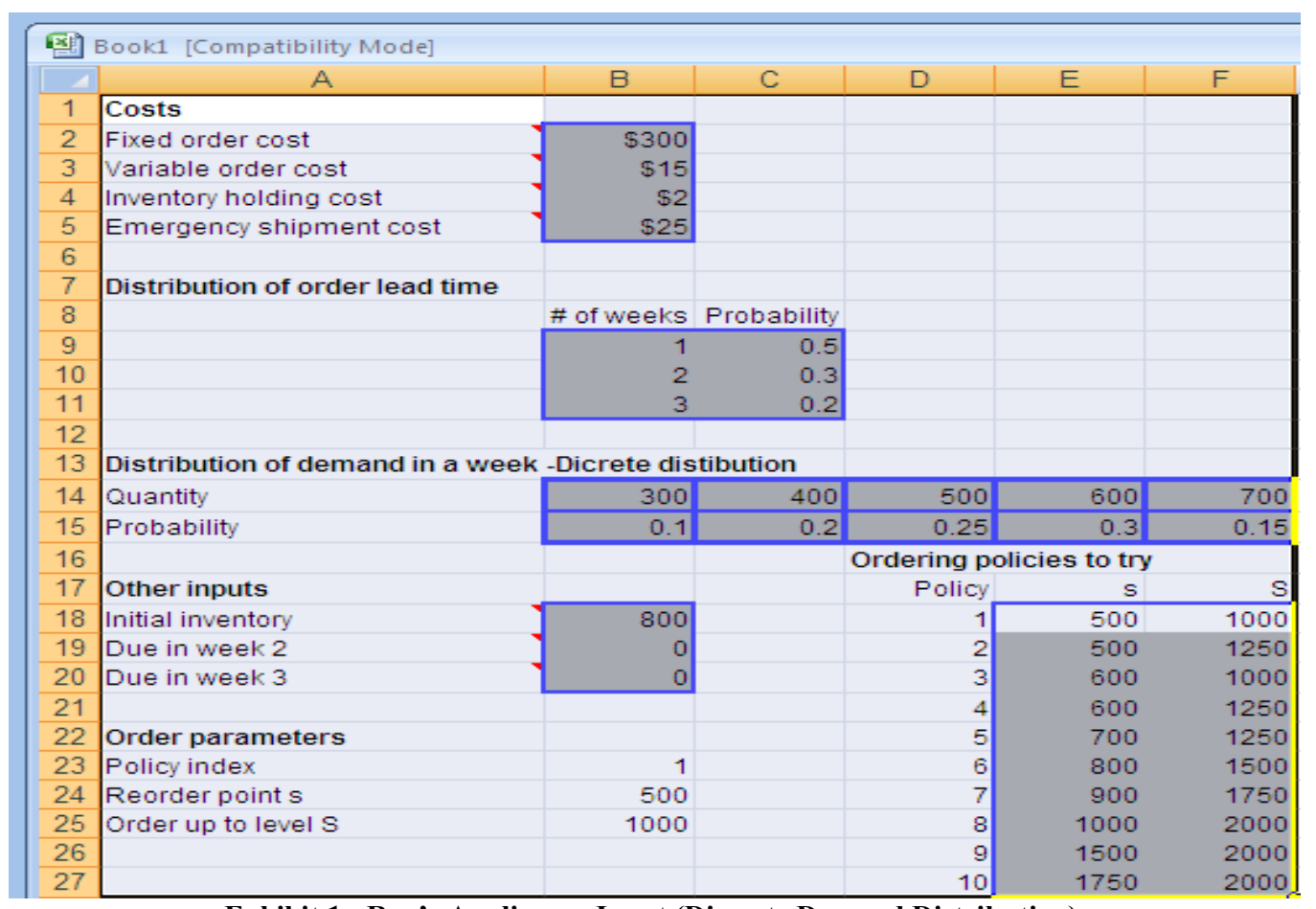

Exhibit 1: Ray's Appliances Input (Discrete Demand Distribution) 
In this example, Palisade @RISK is used to simulate a 52-week period model using ten trial values of (s, S). The spreadsheet model is designed to keep track of the inventory position along with the quantity and timing of arrival of any order. The model is also designed to maintain the record of the fixed cost, variable cost, holding cost and emergency cost incurred in the ordering process over the 52-week period. Exhibit 2 presents the ten simulations summary results for the total inventory cost where total inventory cost is the sum of the fixed cost, variable cost, holding cost and the emergency cost. The number of iterations in each simulation is set to one thousand. Each iteration simulates a fifty-two week period. The model will run ten simulations, one for every combination of the trial values of (s, S). Exhibit 3 illustrates the histogram of the total inventory cost for simulation trial number nine. Appendix A provides the histograms for the total inventory costs for the ten simulations.

\begin{tabular}{|l|l|l|l|l|l|l|l|}
\hline $\begin{array}{c}\text { Simulation } \\
\text { Number }\end{array}$ & S & S & Min & Mean & Max & $\mathbf{5 \%}$ & 95\% \\
\hline 1 & 500 & 1000 & $\$ 490,200$ & $\$ 553,080$ & $\$ 630,200$ & $\$ 517,100$ & $\$ 587,700$ \\
\hline 2 & 500 & 1250 & $\$ 476,700$ & $\$ 547,924$ & $\$ 621,300$ & $\$ 510,950$ & $\$ 583,650$ \\
\hline 3 & 600 & 1000 & $\$ 477,200$ & $\$ 543,507$ & $\$ 630,100$ & $\$ 506,700$ & $\$ 579,200$ \\
\hline 4 & 600 & 1250 & $\$ 484,450$ & $\$ 544,919$ & $\$ 624,700$ & $\$ 508,750$ & $\$ 580,350$ \\
\hline 5 & 700 & 1250 & $\$ 476,350$ & $\$ 539,107$ & $\$ 618,650$ & $\$ 503,700$ & $\$ 574,150$ \\
\hline 6 & 800 & 1500 & $\$ 471,400$ & $\$ 529,894$ & $\$ 601,700$ & $\$ 494,700$ & $\$ 565,300$ \\
\hline 7 & 900 & 1750 & $\$ 468,500$ & $\$ 528,373$ & $\$ 601,100$ & $\$ 496,150$ & $\$ 563,150$ \\
\hline 8 & 1000 & 2000 & $\$ 474,100$ & $\$ 528,993$ & $\$ 595,800$ & $\$ 498,200$ & $\$ 560,300$ \\
\hline $\mathbf{9}$ & $\mathbf{1 5 0 0}$ & $\mathbf{2 0 0 0}$ & $\mathbf{\$ 4 6 8 , 3 0 0}$ & $\mathbf{\$ 5 2 4 , 2 0 7}$ & $\mathbf{\$ 5 8 0 , 7 0 0}$ & $\mathbf{\$ 5 0 0 , 9 0 0}$ & $\mathbf{\$ 5 5 0 , 3 0 0}$ \\
\hline 10 & 1750 & 2000 & $\$ 483,300$ & $\$ 529,242$ & $\$ 574,100$ & $\$ 508,800$ & $\$ 552,200$ \\
\hline
\end{tabular}

Exhibit 2: Summary of Simulation Results for the Total Inventory Cost Using @RISK (Discrete Demand Distribution)

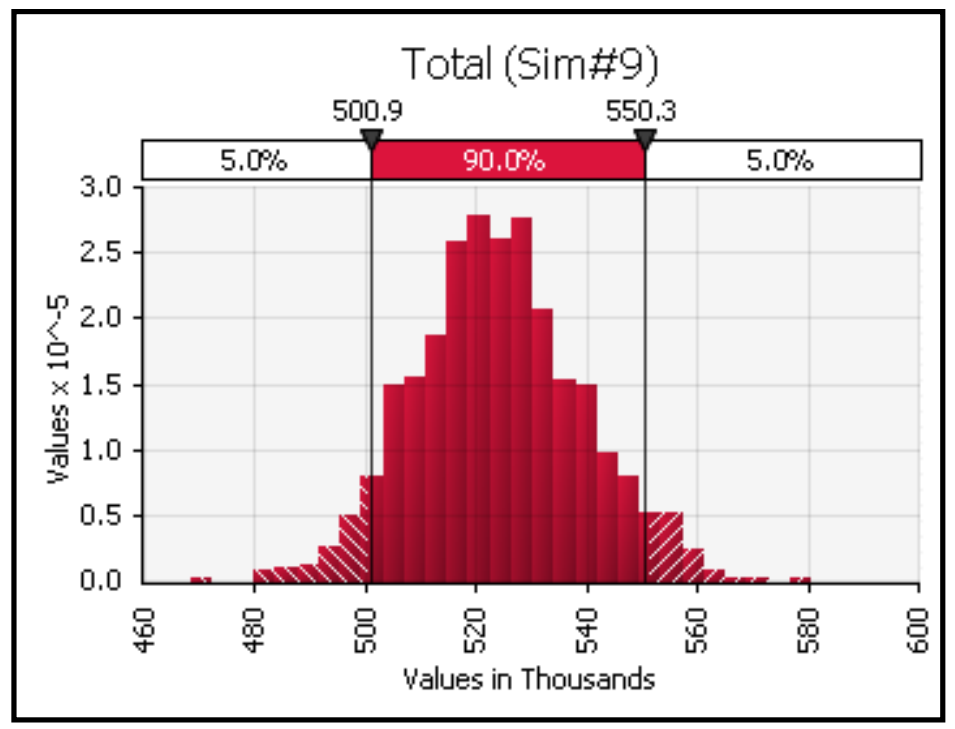

Exhibit 3: Histogram of Total Cost for Simulation \# 9 (Discrete Demand Distribution)

On the other hand, Palisade's RISKOptimizer is used to find the optimal combination of (s, S) that will minimize the average total cost of inventory. Palisade's RISKOptimizer is the simulation optimization add-in for Microsoft Excel that combines the Monte Carlo simulation technology of @RISK with genetic algorithm optimization technology to allow the optimization of Excel spreadsheet models that contain uncertain values. In our numerical example, Ray's Appliances has two uncertain input variables, the lead time and the demand. The variability in the lead time and in the demand is approximated by discrete probability distributions. RISKOptimizer is used to minimize the mean of the total inventory cost by changing the values of $(\mathrm{s}, \mathrm{S})$, where $\mathrm{s}$ is less than or equal to $\mathrm{S}$ taking into considerations the uncertainty in both the demand and lead time. Exhibit 4 presents a summary 
of RISKOptimizer results for the total cost of inventory. Appendix C provides Palisade RISKOptimizer optimization summary results.

\begin{tabular}{|c|c|c|c|c|c|c|}
\hline $\mathbf{S}$ & $\mathbf{S}$ & Min & Mean & Max & $\mathbf{5 \%}$ & $\mathbf{9 5 \%}$ \\
\hline 1000 & 1341 & $\$ 457,462$ & $\mathbf{\$ 5 1 7 , 1 4 7}$ & $\$ 576637$ & $\$ 484,000$ & $\$ 550,100$ \\
\hline
\end{tabular}

Exhibit 4: Summary of RISKOptimizer Results for the Total Inventory Cost (Discrete Demand Distribution)

\section{DISCUSSION OF THE RESULTS FOR EXAMPLE 1}

The strength of @RISK is that it keeps track of any output the modeler specifies and then allows the modeler to show the corresponding results as graphs or tables in temporary or permanent worksheets. Many discussions of simulation results focus on the mean of some output variable. This makes sense in our study, given the importance of the Expected Monetary Value (EMV) approach for decision making. The Expected Monetary Value approach is most appropriate when a decision maker is neither risk-averse nor risk-seeking, but is risk-neutral. Typically, well-established organizations with numerous decisions of this nature tend to use expected value because it provides an indication of the long-run average payoff or cost. The EMV for any decision is a weighted average of the possible payoffs or costs for this decision. However, analysts are also interested in the extreme values of an output distribution. In this case, modelers can investigate the $5^{\text {th }}$ and the $95^{\text {th }}$ percentiles for the specific output. The $5^{\text {th }}$ percentile is also called Value at Risk (VAR) and it is often used in financial models.

After running ten simulations using @RISK and monitoring the total cost of inventory as an output, simulation number nine provided the lowest mean $(\$ 524,207)$ of the total inventory cost. Its $5^{\text {th }}$ percentile $(\$ 500,900)$ is close to the smallest. It has also provided the lowest $95^{\text {th }}$ percentile $(\$ 550,300)$. It indicates that $95 \%$ of all the times, the total cost of inventory will be less than or equal $\$ 550,300$. Therefore, the final recommendation for Ray's Appliances is to use the trial values of simulation number nine, namely, $(1500,2000)$ for their periodic inventory ordering policy.

When applying Palisade's RISKOptimizer using Ray's Appliances input, the model solved for the optimal values of $(\mathrm{s}, \mathrm{S})$ that will minimize the mean total cost of inventory. Those values are $(1000,1341)$. Using this optimal solution, the mean of total cost is $\$ 517,147$, the $5^{\text {th }}$ percentile is $\$ 484,000$, and the $95^{\text {th }}$ percentile is $\$ 550,100$.

By comparing the two methods, RISKOptimizer offered a very efficient and economic way to solve for the parameters of the periodic inventory policy. The savings in the mean total cost is $\$ 7060$, the $5^{\text {th }}$ percentile is reduced by $\$ 16,900$, and the $95^{\text {th }}$ percentile is lowered by $\$ 200$.

\section{NUMERICAL EXAMPLE 2}

In this example, Ray's Appliances manager is using the same data input from example 1. The only difference will be in the demand behavior. Based on historical data, Ray's Appliances manager approximates the demand to follow a normal distribution with a mean of 600 units and a standard deviation of 150 units.

Ray's Appliances manager is planning to use Palisade @RISK simulation to simulate ten combinations of $(\mathrm{s}, \mathrm{S})$ ordering policy in order to find out which combination provides the lowest cost of inventory over the 52-week period. Exhibit 5 presents Ray's Appliances inputs used in the spreadsheet model for a normal distribution demand, Exhibit 6 presents the ten trial simulations summary results for the total inventory cost, and Exhibit 7 illustrates the histogram of the total cost for simulation number ten. Appendix B provides the histograms for the total inventory costs for the ten simulations. 


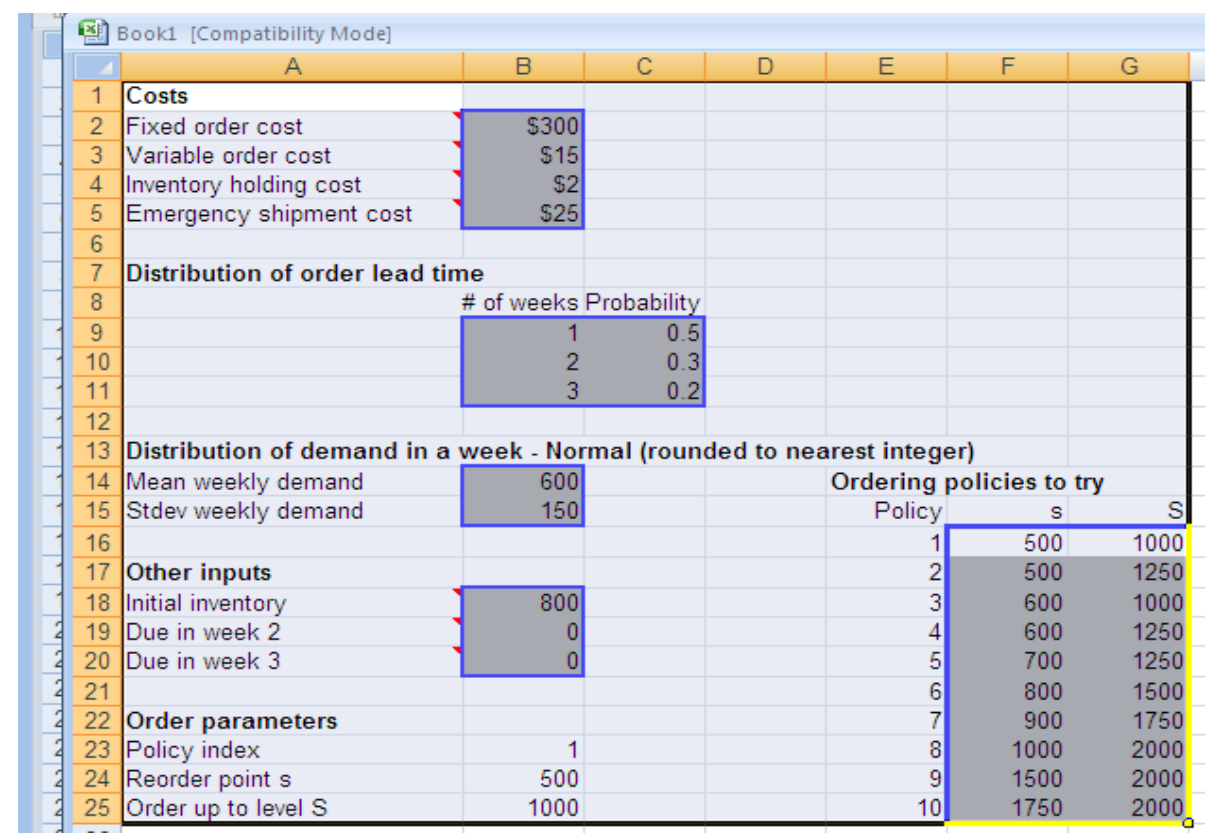

Exhibit 5: Ray's Appliances Input (Normal Demand Distribution)

\begin{tabular}{|c|c|c|c|c|c|c|c|}
\hline Simulation Number & S & S & Min & Mean & Max & $\mathbf{5 \%}$ & $\mathbf{9 5 \%}$ \\
\hline 1 & 500 & 1000 & $\$ 570,747$ & $\$ 652,987$ & $\$ 752,539$ & $\$ 607,602$ & $\$ 697,359$ \\
\hline 2 & 500 & 1250 & $\$ 554,062$ & $\$ 642,710$ & $\$ 735,921$ & $\$ 598,013$ & $\$ 688,023$ \\
\hline 3 & 600 & 1000 & $\$ 552,151$ & $\$ 644,873$ & $\$ 742,869$ & $\$ 599,138$ & $\$ 689,366$ \\
\hline 4 & 600 & 1250 & $\$ 554,062$ & $\$ 640,016$ & $\$ 734,249$ & $\$ 594,430$ & $\$ 685,520$ \\
\hline 5 & 700 & 1250 & $\$ 551,398$ & $\$ 633,548$ & $\$ 718,160$ & $\$ 587,929$ & $\$ 677,797$ \\
\hline 6 & 800 & 1500 & $\$ 537,265$ & $\$ 624,029$ & $\$ 732,839$ & $\$ 580,129$ & $\$ 668,014$ \\
\hline 7 & 900 & 1750 & $\$ 537,821$ & $\$ 617,758$ & $\$ 727,421$ & $\$ 574,068$ & $\$ 660,521$ \\
\hline 8 & 1000 & 2000 & $\$ 545,350$ & $\$ 613,773$ & $\$ 723,017$ & $\$ 572,344$ & $\$ 654,790$ \\
\hline 9 & 1500 & 2000 & $\$ 525,714$ & $\$ 595,704$ & $\$ 672,901$ & $\$ 563,254$ & $\$ 631,170$ \\
\hline 10 & 1750 & 2000 & $\$ 536,144$ & $\$ 592,819$ & $\$ 648,506$ & $\$ 562,305$ & $\$ 626,024$ \\
\hline
\end{tabular}

Exhibit 6: Summary of Simulation Results for the Total Inventory Cost Using @ RISK (Normal Demand Distribution)

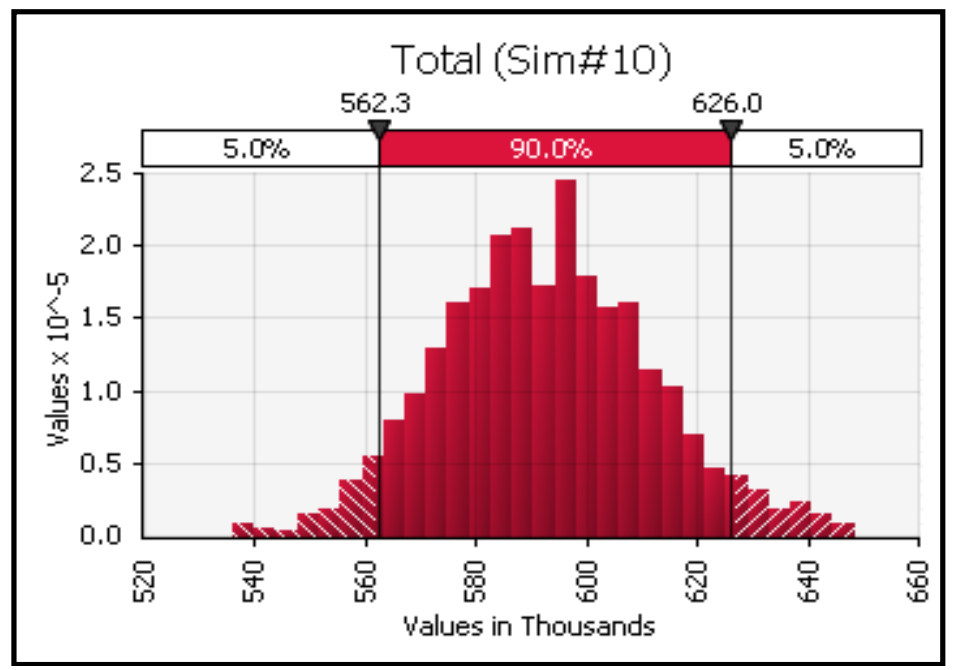

Exhibit 7: Histogram of Total Cost for Simulation \#10 (Normal Demand Distribution) 
On the other hand, Palisade's RISKOptimizer is used to find the optimal combination of (s, S) that will minimize the average total cost of inventory. Exhibit 8 presents a summary of RISKOptimizer results for the total cost of inventory using a normal distribution for the demand. Appendix D provides Palisade RISKOptimizer optimization summary results.

\begin{tabular}{|c|c|c|c|c|c|c|}
\hline $\mathbf{S}$ & $\mathbf{S}$ & Min & Mean & Max & $\mathbf{5 \%}$ & $\mathbf{9 5 \%}$ \\
\hline 1310 & 1568 & $\$ 525,075$ & $\mathbf{\$ 5 9 2 , 4 1 5}$ & $\$ 661,698$ & $\$ 560,991$ & $\$ 625,263$ \\
\hline
\end{tabular}

Exhibit 8: Summary of RISKOptimizer Results for the Total Inventory Cost (Normal Demand Distribution)

\section{DISCUSSION OF THE RESULTS FOR EXAMPLE 2}

After running ten simulations using @Risk and monitoring the total cost of inventory as an output, simulation number ten has provided the lowest mean $(\$ 592,819)$ of the total inventory cost. It has also displayed the smallest $5^{\text {th }}(\$ 562,305)$ and $95^{\text {th }}(\$ 626,024)$ percentiles. Therefore, the final recommendation for Ray's Appliances is to use the trial values of simulation number ten, namely, $(1750,2000)$ for their periodic inventory ordering policy.

When applying Palisade's RISKOptimizer using Ray's Appliances input, the model solved for the optimal values of $(\mathrm{s}, \mathrm{S})$ that will minimize the mean total cost of inventory. Those values are $(1310,1568)$. Using this optimal solution, the mean of total cost is $\$ 592,415$, the $5^{\text {th }}$ percentile is $\$ 560,991$ and the $95^{\text {th }}$ percentile is $\$ 625,263$.

By comparing the two methods, RISKOptimizer offered one more time a very efficient and economic way to solve for the parameters of the periodic inventory policy. The savings in the mean total cost is $\$ 404$, the $5^{\text {th }}$ percentile is reduced by $\$ 1314$, and the $95^{\text {th }}$ percentile is lowered by $\$ 761$.

\section{CONCLUSION}

Managing uncertain inventories is an important topic that has received attention from academics and managers because of its major effect on the economic performance of the firm. One of the main roles of inventory managers is to face the challenge of keeping inventory levels as low as possible without increasing overall costs or negatively impacting the product availability. Due to the difficulty of dealing with the uncertainties of demand and lead time, this study provides a simulation-based model that allows managers to combine assumptions about uncertain demand and ordering policies and then play out the events as they occur through time.

In this paper, Palisade @Risk simulation is used to determine the value of $(\mathrm{s}, \mathrm{S})$ in a periodic review inventory model with uncertain demand and lead time. A periodic review inventory of 52 weeks is illustrated in a spreadsheet model in order to minimize the expected cost of inventory per week. This paper provided a comparison between the use of Palisade @RISK simulation with trial values of $(\mathrm{s}, \mathrm{S})$ and the use of Palisade RISKOptimizer to find optimal value of $(\mathrm{s}, \mathrm{S})$ that minimize the expected cost of inventory in a periodic review model. RISKOptimizer combines the genetic algorithm technology of Evolver with the Monte Carlo simulation engine of @ RISK to solve optimization problems. It is capable of running multiple simulations to find a better set of parameters to optimize simulation results.

The spreadsheet models in this paper will provide inventory managers with a technique to use in order to minimize total inventory cost. It will also allow managers to make changes to the model as the changes occur. Managers can change the approximation to the probability distributions of demand and lead time when getting access to more historical data. Using RISKOptimizer, managers can solve for the optimal values of (s, S) without thinking of what trial values to use. Sometimes trial values can be far away from the optimal solution. The major advantage of using simulation to deal with uncertainty is that it does not require managers to have mathematical skills to apply it. Despite the fact that dealing with uncertainty requires a great deal of understanding how to logically build the model, the use of simulations brings such complicated models within the grasp of managers who are not mathematicians. 


\section{AUTHOR INFORMATION}

Raida Abuizam is an Associate Professor of Operations Management in the Department of Finance, Economics and Quantitative Methods at Purdue University Calumet, Hammond Indiana, U.S.A. She has a Ph.D. from Illinois Institute of Technology in Management Science/Operations Management and has published in the Journal of Business and Economic Research, Southwestern Business Administration Journal, and Journal of Business Case Studies. She can be reached at abuizam@ calumet.purdue.edu.

\section{REFERENCES}

1. Barankin, E.W. (1961), "A delivery-lag inventory model with an emergency provision," Naval Research Logistics, Vol.8, pp 285-311.

2. Bashyam, S. and Fu, M.C. (1998), "Optimization of (s, S) inventory systems with random lead times and a service level constraint," Management Science, Vol.44, pp 243-256.

3. Blumenfield, D.E., Hall, R.W. and Jordan, W.C. (1985), "Tradeoff between freight expediting and safety stock inventory costs," Journal of Business Logistics, Vol.6, pp 79-100

4. Bulinskaya, E. (1964), "Some results concerning optimal inventory policies," Theory of probability and applications, Vol.9, pp 502-507

5. Chang, C. and Gutierrez, G.J. (1996), "A periodic review inventory system with two supply modes," European Journal of operational Research, Vol.94, pp 389-403

6. Chang, C. and Gutierrez, G.J. (1998), "Optimal control policies for a periodic review inventory system with emergency orders," Naval Research Logistics, Vol.45, pp 187-204

7. Daniel, K.H. (1962), "A delivery-lag inventory model with emergency, in multistage inventory model and techniques," Stanford University Press, Stanford, CA, pp

8. $\quad 32-46$

9. Gross, D. and Soriano, A. (1972), "On the economic application of airlift to product distribution and its impact on inventory levels," Naval Research Logistics, Vol.19, pp 501-507

10. Lee, H.L. and Nahmias, S. (1993), "Single-product, single location models," Handbook on Operations Research and Management Science, Vol.4, pp 192-198

11. Neuts, M. F. (1964), "An inventory model with optional lag time," SIAM Journal of Applied Mathematics, Vol.12, pp 179-185

12. Schneider, H. and Ringuest, J.L. (1990), "Power approximation in computing (s, S) policies using service level," Management Science, Vol.36, pp 822-834

13. Tagaras, G. and Vlachos, D. (2001), "A periodic review inventory system with emergency replenishments," Management Science, Vol.47, pp 415-429

14. Tims, H.C. and Groenevelt, H. (1984), "Simple approximations for the reorder point in periodic and continuous review (s, S) inventory systems with service level constraints," European Journal of Operational Research, Vol.17, pp175-190

15. Veinnot, A.F. Jr. (1966), "The status of mathematical inventory theory,” Management Science, Vol.12, pp 745-777

16. Whittemore, A.S. and Saunders, S. (1977), “Optimal inventory under stochastic demand with two supply options," SIAM Journal of Applied Mathematics, Vol.32, pp 293-305

17. Winston, W., and Albright, S. C. (2007), Practical Management Science, third edition, Duxbury

18. Wright, G.P. (1968), "Optional policies for a multi-product inventory system with negotiable lead times," Naval Research Logistics, Vol.15, pp 375-401. 


\section{APPENDIX A}

Histogram of Total Cost of Inventory (Discrete Demand Distribution)
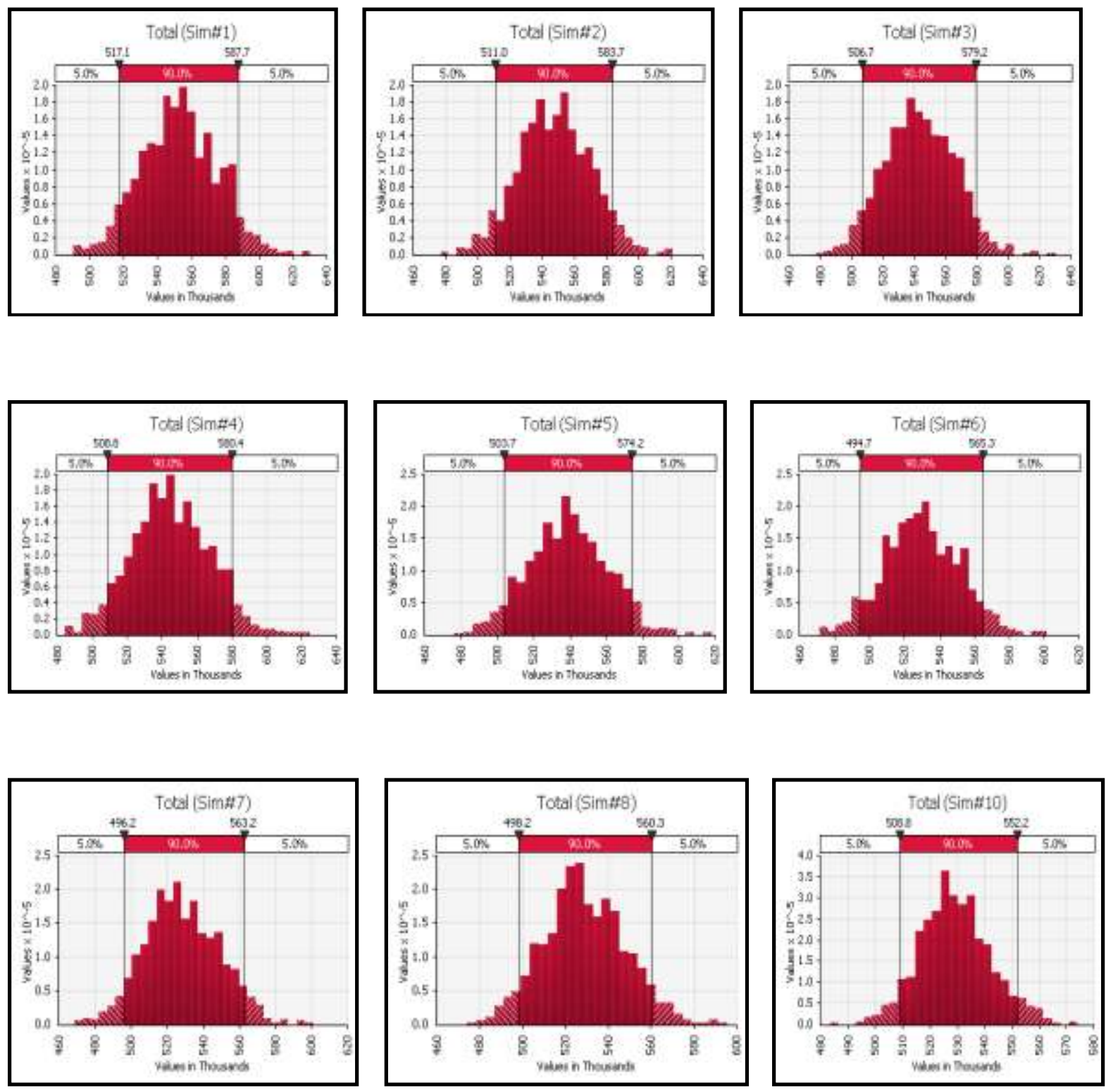


\section{APPENDIX B}

Histogram of Total Cost of Inventory (Normal Demand Distribution)
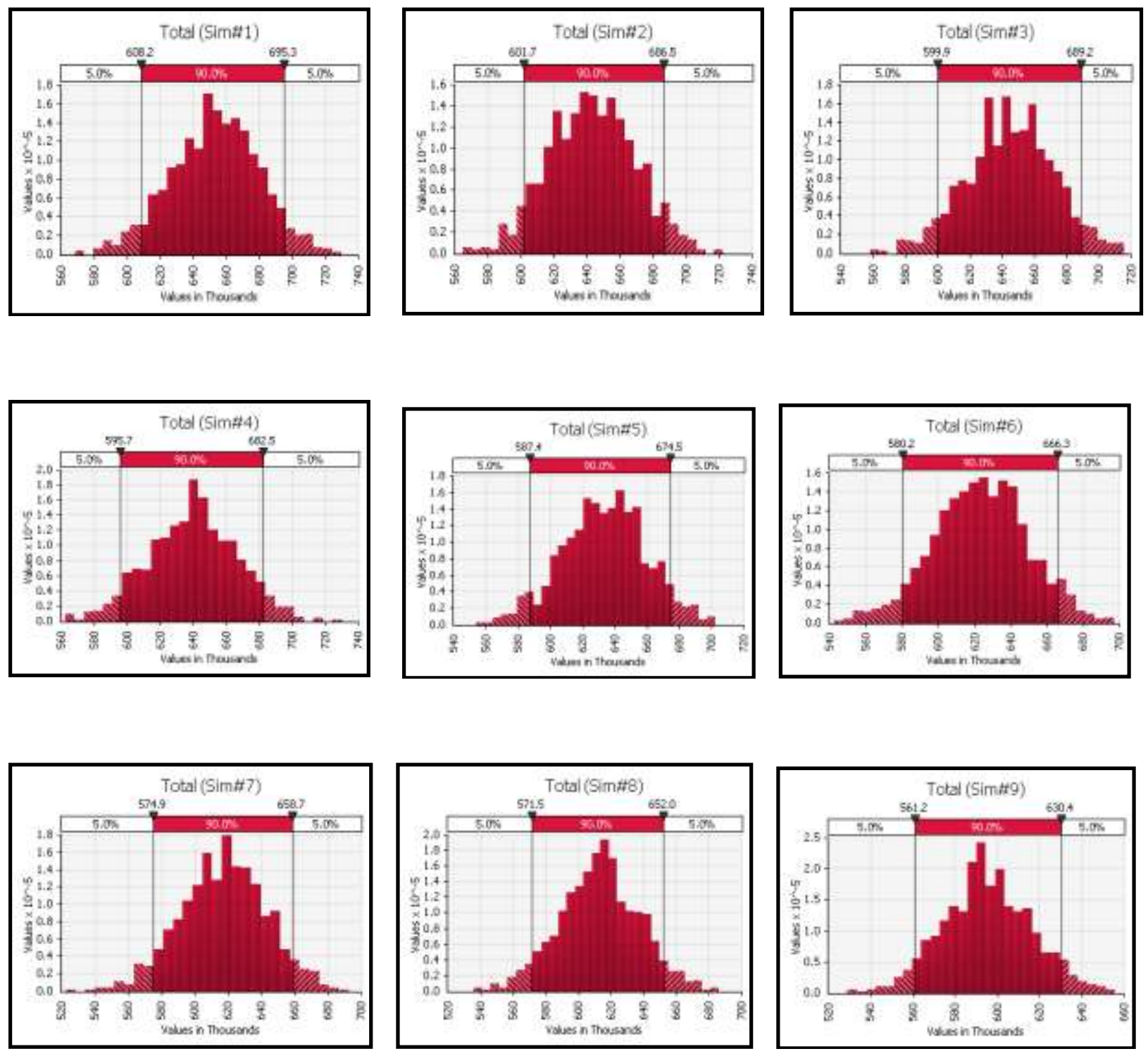


\section{APPENDIX C}

\section{RISKOptimizer Summary of Result (Discrete Demand Distribution)}

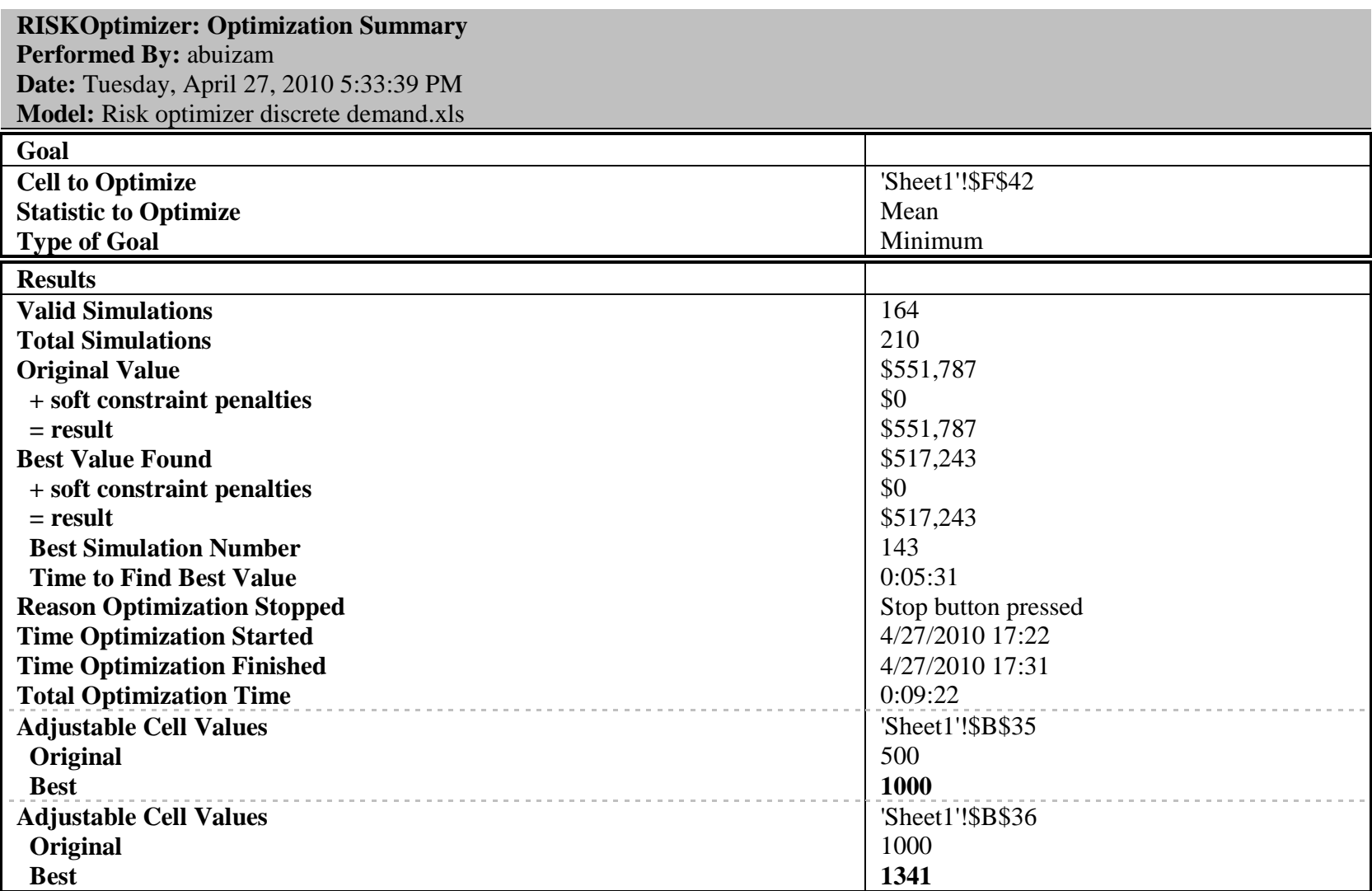




\section{APPENDIX D}

\section{RISKOptimizer Summary of Result (Normal Demand Distribution)}

RISKOptimizer: Optimization Summary

Performed By: abuizam

Date: Tuesday, April 27, 2010 4:28:47 PM

Model: Risk optimizer Normal demand.xls

\begin{tabular}{|c|c|}
\hline Goal & \\
\hline Cell to Optimize & 'Sheet1'!\$F\$42 \\
\hline Statistic to Optimize & Mean \\
\hline Type of Goal & Minimum \\
\hline Results & \\
\hline Valid Simulations & 129 \\
\hline Total Simulations & 148 \\
\hline Original Value & $\$ 605,206$ \\
\hline + soft constraint penalties & $\$ 0$ \\
\hline$=$ result & $\$ 605,206$ \\
\hline Best Value Found & $\$ 592,415$ \\
\hline+ soft constraint penalties & $\$ 0$ \\
\hline$=$ result & $\$ 592,415$ \\
\hline Best Simulation Number & 129 \\
\hline Time to Find Best Value & $0: 09: 55$ \\
\hline Reason Optimization Stopped & Stop button pressed \\
\hline Time Optimization Started & $4 / 27 / 201016: 13$ \\
\hline Time Optimization Finished & $4 / 27 / 201016: 28$ \\
\hline Total Optimization Time & $0: 13: 20$ \\
\hline Adjustable Cell Values & 'Sheet1'!\$B $\$ 35$ \\
\hline Original & 997 \\
\hline Best & 1310 \\
\hline Adjustable Cell Values & 'Sheet1'! $\$$ B $\$ 6$ \\
\hline Original & 1365 \\
\hline Best & 1568 \\
\hline
\end{tabular}


International Journal of Management \& Information Systems - First Quarter $2011 \quad$ Volume 15, Number 1 NOTES 